\title{
The Role of P2 Receptor-Mediated Component in Neurogenic Tone Control of Human Great Saphenous Vein
}

DOI: 10.17691/stm2017.9.1.10

Received March 2, 2016

B.A. Ziganshin, MD, Assistant, Department of Surgical Diseases No.21;

L.R. Giniyatova, MD, PhD, Assistant, Department of Pediatrics';

D.A. Slavin, MD, PhD, Associate Professor, Department of Endoscopy, General and Endoscopic Surgery²;

R.R. Kamaliev, PhD, Assistant, Department of Pharmacology with Courses of Pharmacognosy and Botany';

A.P. Ziganshina, MD, PhD Student, Department of Ophthalmology';

A.A. Spasov, MD, DSc, Professor, Academician of the Russian Academy of Sciences, Head of the Department of Pharmacology;

R.K. Dzhordzhikiya, MD, DSc, Professor, Head of the Department of Surgical Diseases No.2;

A.U. Ziganshin, MD, DSc, Professor, Head of the Department of Pharmacology with Courses

of Pharmacognosy and Botany'; Professor, Department of Fundamental and Clinical Pharmacology ${ }^{4}$

${ }^{1}$ Kazan State Medical University, 49 Butlerov St., Kazan, Republic of Tatarstan, 420012, Russian Federation;

${ }^{2}$ Kazan State Medical Academy, 36 Butlerov St., Kazan, Republic of Tatarstan, 420012, Russian Federation;

${ }^{3}$ Volgograd State Medical University, 1 Pavshikh Bortsov Square, Volgograd, 400008, Russian Federation;

${ }^{4}$ Kazan (Volga region) Federal University, 18 Kremlevskaya St., Kazan, Republic of Tatarstan, 420008,

Russian Federation

The aim of the investigation was to evaluate experimentally the role of the $\mathrm{P} 2$ receptor-mediated component in neurogenic tone control of human varicose-affected and healthy great saphenous vein (GSV).

Materials and Methods. The material for the study were segments of GSV obtained from two groups of patients: group 1 ( $n=14$ ) included patients with varicose vein disease, in whom GSV was removed during surgical treatment; group 2 ( $n=21)$ comprised patients with coronary artery disease who underwent coronary artery bypass grafting using GSV as an autograft. Mechanical activity of the isolated veins was studied in vitro by electrical stimulation before and after incubation with atropine and phentolamine, pyridoxal-phosphate-6-azophenyl2',4'-disulfonate (PPADS) and suramin (both nonselective antagonists of P2 receptors), and also after desensitization of P2X receptors by $\alpha, \beta$-methylene ATP.

Results. Atropine and phentolamine did not completely inhibit the contractile responses evoked by electric field stimulation of the varicose and non-varicose GSV. PPADS $(10$ and $30 \mu \mathrm{M})$ and suramin $(100$ and $300 \mu \mathrm{M})$ significantly reduced the contractile amplitude of GSV response in both groups of veins $(p<0.05)$ in the presence of atropine and phentolamine. $\alpha, \beta$-methylene ATP $(10 \mu M)$ did not significantly reduce the amplitude of the GSV contractile response $(p>0.05)$. Atropine, phentolamine and PPADS inhibited the contractile responses to a lesser extent in the varicose-affected veins compared to the veins unaffected by varicose disease.

Conclusion. These experimental results suggest the presence of the P2 receptor-mediated component in the neurogenic control of human GSV tone. Further studies of the P2 receptor mechanism of action is promising for the development of drugs both for treating varicose veins and for preventing spasm of the venous grafts after aortocoronary bypass surgery.

Key words: P2 receptors; human great saphenous vein; GSV; varicose vein disease.

Over several recent decades, the presence of P2 receptors has been found in many blood vessels of humans and animals [1]. This is a special class of receptors the endogenous ligand for which is adenosine triphosphate (ATP) [2]. P2 receptors are divided into two large families - $\mathrm{P} 2 \mathrm{X}$ and $\mathrm{P} 2 \mathrm{Y}$ receptors, which are

For contacts: Ayrat U. Ziganshin, e-mail: auziganshin@gmail.com 
different in their structure and mechanism of activation. P2X receptors represent typical ligand-operating ion channels, while $\mathrm{P} 2 \mathrm{Y}$ receptors are metabotropic G protein-mediated receptors [3]. P2X receptors located on smooth muscle cells mediate vasoconstrictor action of nucleotides on the vessels, whereas endothelial P2Y receptors often provide vasodilatation [4]. At present, data on the role of extracellular ATP in the regulation of the human cardiovascular system as a whole and in blood vessels in particular are being accumulated [1]. Recent investigations provide evidence regarding the potential of using agonists and antagonists of P2 receptors in clinical practice [5].

The human great saphenous vein (GSV) is an important blood vessel, since it is most frequently used as an autograft in various bypass operations (aortocoronary bypass, femoral popliteal bypass, etc.). At the same time, the GSV is most commonly affected by varicose disease of the lower extremities caused by chronic venous disease (CVD, previously called chronic venous insufficiency) [6], that limits its suitability for surgical procedures.

Availability and activity of $\mathrm{P} 2$ receptors in human GSV have been shown in our previous studies [7-10]. However, the physiological and pathophysiological role of these receptors in the human GSV affected and non-affected by varicose disease remains unclear. In particular, it is unknown whether $\mathrm{P} 2$ receptors located in the GSV participate in formation of the vascular wall tone and whether they are activated in response to the nerve stimuli.

The aim of the investigation was to study experimentally the involvement of P2 receptors in realizing the neurogenic tone control of the human great saphenous vein affected and non-affected by varicose disease.

\section{Materials and Methods}

Protocol of tissue collection for the experiments. Segments of the GSV, which were obtained from the patients during surgical interventions requiring GSV specimen collection, were used in the study. All patients underwent surgical treatment in the hospitals of Kazan city. Patients were divided into two groups.

Group 1 included patients with varicose disease of the lower extremities at the subcompensation stage (stage C2-C3 in compliance with the CEAP classification [11]), who underwent phlebectomy according to TroyanovTrendelenburg-Babcock. The group consisted of 14 patients: 12 females and 2 males at the age of 32-60 years. A $1.5-2 \mathrm{~cm}$ long segment of the proximal portion of the GVS was collected from every patient from the upper third of the thigh approximately $2-3 \mathrm{~cm}$ away from the saphenofemoral junction. This GSV segment was the most convenient for intraoperative collection and provided minimal traumatization of the blood vessel, ensuring viability of the tissue throughout the experiment duration.

Group 2 comprised patients with ischemic heart disease, exertional angina, functional class III-IV; postinfarction cardiosclerosis was present in the majority of patients. This group of patients underwent aortocoronary bypass using the GSV as an autograft. The group consisted of 21 patients: 20 males and 1 female at the age of $41-65$ years. A vein segment $1-1.5 \mathrm{~cm}$ long was collected within the upper third of the thigh, as it is in this area that the GSV is used most often for aortocoronary bypass procedures. Ultrasound examination of the GSV of both lower extremities was performed in each patient of this group at the preoperative stage to reveal pathological dilatation and retrograde flow. Only patients with normal findings on the ultrasound examination (total lack of retrograde flow) and without other signs of chronic venous insufficiency were included in the study.

Preparation of vascular specimens for the experiment. Immediately after intraoperative GSV collection, the vein segment intended for the experiment, was placed in the modified Krebs solution (cooled to $4^{\circ} \mathrm{C}$ ) and transported within a 120-min period to the pharmacological laboratory for experimentation. The modified Krebs solution (in $\mathrm{mM}$ ) was composed of $133 \mathrm{NaCl} ; 4.7 \mathrm{KCl} ; 16.3 \quad \mathrm{NaHCO}_{3} ; 0.6 \mathrm{MgSO}_{4}$; $1.35 \mathrm{NaH}_{2} \mathrm{PO}_{4} ; 2.5 \mathrm{CaCL}_{2} ; 7.8$ glucose. Afterward, the GVS segment was dissected and prepared to be used in the pharmacological experiment.

The experiments were carried out using a pharmacological unit (Linton Instrumentation, Great Britain) for registration of mechanical activity of isolated tissues (Figure 1). The vessels were thoroughly and carefully cleaned from blood, adjacent tissues were removed. Ring-shaped segments $1-2 \mathrm{~cm}$ in width were cut from the GSV specimen, the rings were then cut open to obtain a smooth-muscle specimen $8-10 \mathrm{~cm}$ long, and a silk thread was tied to its each end. The circular smooth muscle layer in the specimen was located longitudinally. These specimens were stretched between the two rings of platinum electrodes (used for electrical stimulation of the tissue), one thread was fixed and the other one was connected to the FSG-01 isometric sensor of mechanical activity (Linton Instrumentation, Great Britain). Then the specimen with the electrode was placed into a constant-temperature bath, containing $10 \mathrm{ml}$ of modified Krebs solution. The temperature of the solution in the bath was maintained constantly at $37.0 \pm 0.5^{\circ} \mathrm{C}$. The solution was continuously aerated by the gas mixture consisting of oxygen (95\%) and carbon dioxide (5\%) to keep the $\mathrm{pH}$ level at 7.3-7.4. Mechanical activity of the blood vessel was recorded using a personal computer and the MP100WSW Data Acquisition System (BIOPACK Systems Inc., USA).

Prior to the beginning of the experiment, a 1-g load was applied to the specimens, and in such a state the vessel was allowed to equilibrate for $60 \mathrm{~min}$ during which the Krebs solution in the baths was changed 4 times. Further, mechanical activity of the isolated 


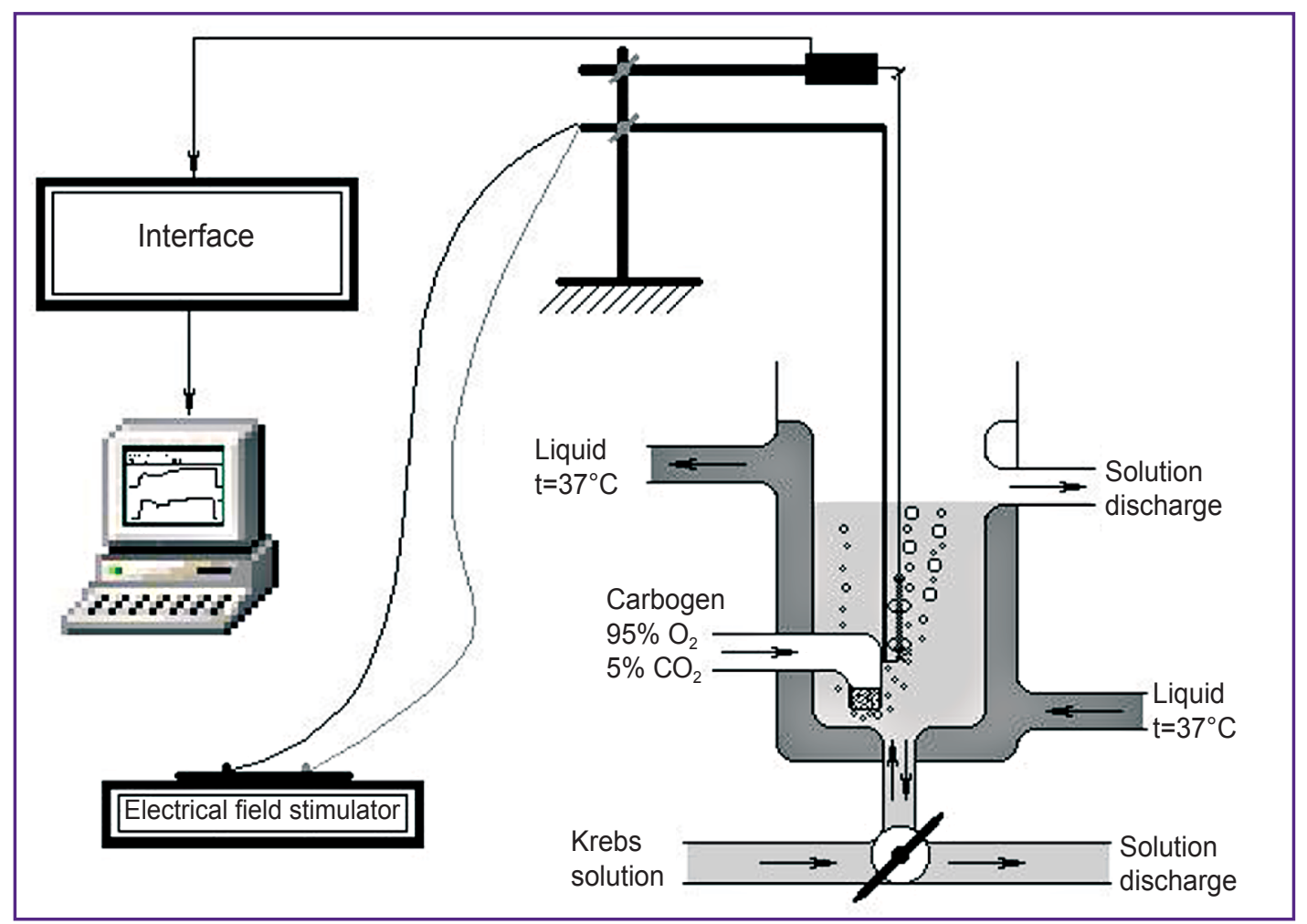

Figure 1. Schematic presentation of the pharmacological organ-bath unit used for the experiments

GSV segments was registered in response to electrical stimulation before and after introduction of agonists or antagonists into the organ-bath.

Protocol of electrical stimulation of the vascular specimens. In order to study the neurogenic control of the GSV tone and detect the P2 receptor component of the contraction, the tissue response to electrical stimulation ( $4 \mathrm{~Hz}, 20 \mathrm{~V}$ ) was evaluated before and after incubation with M-cholinoblocker, atropine $(0.1 \mu \mathrm{M})$, in combination with an a-adrenoblocker, phentolamine $(1 \mu \mathrm{M})$, antagonists of P2 receptors, pyridoxalphosphate6-azophenyl-2',4'-disulfonic acid (PPADS) (10 and $30 \mu \mathrm{M})$ and suramin $(100$ and $300 \mu \mathrm{M})$, as well as by means of $\mathrm{P} 2 \mathrm{X}$ receptor desensitization with a $\mathrm{P} 2 \mathrm{X}$ receptor agonist, $\alpha, \beta$-methylene ATP $(10 \mu \mathrm{M})$, which was chosen as the most effective and enzymatically stable analog of ATP. Digitimer D330 MultiStim system (United Kingdom) was used for electric stimulation. The initial contractile response was taken as $100 \%$ and was registered before incubation with any antagonists. Further, the antagonists were added into the bath and the effect of electrical stimulation was assessed again (Figure 2 (a)). Once the maximal effect from stimulation was achieved, the specimens were washed several times with the Krebs solution and after a time interval of no less than 30 min the next antagonist was introduced. In each case, control electrical stimulation, which caused equal contraction of the vessel throughout the experiment, was performed without addition of any agonists or antagonists into the organ-bath (Figure 2 (b)). Electrical stimulation parameters were selected in such a way as to stimulate the nerve terminals only, rather

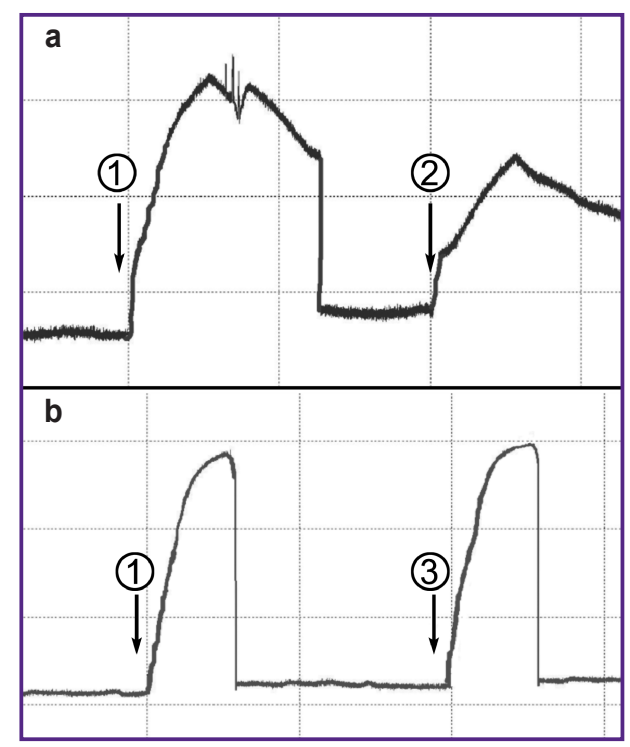

Figure 2. Mechanical tracings obtained during electrical stimulation of the great saphenous vein: (a) tracing of GVS's contractile responses evoked by electrical stimulation before (1) and after incubation with PPADS (2); (b) control tracing of GVS's contractile responses evoked by electrical stimulation $(1,3)$ 
than the smooth muscles. To ascertain the neurogenic origin of the GSV contractile response to electrical field stimulation, the control specimens were incubated with a selective blocker of sodium channels, tetrodotoxin $(1 \mu \mathrm{M})$, and the contractile response before and after incubation was evaluated.

The compounds studied. Atropine sulfate, phentolamine, PPADS, suramin sodium salt, $\alpha, \beta-$ methylene ATP, and tetrodotoxin were supplied by Sigma-Aldrich Corporation, USA.

Statistical data processing. The results were analyzed using the Graph Pad Prism statistical program (USA). The Student's t-test for correlated and independent samples, and one-way analysis of variance (ANOVA) were also used. The difference was considered statistically significant at $p<0.05$.

Ethical aspects. This investigation was approved by the Ethics Committee of Kazan State Medical University. Informed consent for participation in the study was obtained from every patient.

Results. Investigations of neurogenic control of the GSV tone showed that a combined application of atropine $(0.1 \mu \mathrm{M})$ and phentolamine $(1 \mu \mathrm{M})$ did not inhibit completely the contractile responses caused by electric field stimulation. This effect was observed both in varicose and non-varicose GSV specimens. In varicose veins atropine and phentolamine inhibited the effect of electrostimulation by $30.5 \pm 4.9 \%$, whereas in non-varicose GSV by $38.0 \pm 3.3 \%$. Antagonist of P2 receptors, PPADS, at concentration of $10 \mu \mathrm{M}$ statistically significantly decreased the amplitude of contractile response in the presence of atropine and phentolamine in varicose-affected and non-affected GSV by $47.9 \pm 6.0 \%$ $(p=0.046)$ and $51.9 \pm 3.9 \%(p=0.013)$, respectively. No dependence of the GSV response was observed on the decrease of the PPADS concentration, as at $30 \mu \mathrm{M}$ the antagonistic effect of PPADS did not significantly differ from the one at $10 \mu \mathrm{M}(\mathrm{p}=0.63$ for varicose GSV and $p=0.24$ for GSV unaffected by varicose disease) (Figure 3).

A similar situation was observed when another nonselective antagonist of $\mathrm{P} 2$ receptors, suramin, was studied. Suramin statistically significantly reduced the GSV contractile response by $46.3 \pm 2.6 \% \quad(p=0.030)$ in varicose veins and by $51.7 \pm 3.3 \%(p=0.043)$ in GSV segments not affected by varicose disease at the concentration of $100 \mu \mathrm{M}$, whereas further increase of its concentration to $300 \mu \mathrm{M}$ did not reduce the contractile amplitude (Figure 4).

P2X receptor agonist, $\alpha, \beta$-methylene ATP, capable of desensitizing $\mathrm{P} 2 \mathrm{X}$ receptors after prolonged exposure, at a concentration of $10 \mu \mathrm{M}$ decreased insignificantly the amplitude of the GSV contractile responses in both groups: by $39.9 \pm 2.6 \%$ for varicose veins and by $42.8 \pm 8.7 \%$ for non-varicose GSV, but in this case no statistical significance was established $(p=0.250$ and $p=0.532$, respectively) (Figure 5).

The following results were obtained when varicose veins were compared to non-varicose ones. Atropine, phentolamine and PPADS inhibited contractions to a lesser extent in the group of varicose-affected veins compared to the veins non-affected by varicose disease. Such dependence was established neither for suramin nor for $\alpha, \beta$-methylene ATP.

In the experiments with tetrodotoxin $(1 \mu \mathrm{M})$ we have confirmed that the obtained contractile responses are definitely of neurogenic origin, i.e. under the action of the electrical field only nerve terminals are stimulated and not the muscular components of the vascular wall. After incubation with tetrodotoxin, electrical field stimulation no longer evoked contractile responses of the GVS, which is explained by the blockage of sodium channels of the nerve terminals resulting in conduction blockage of the nervous impulses.

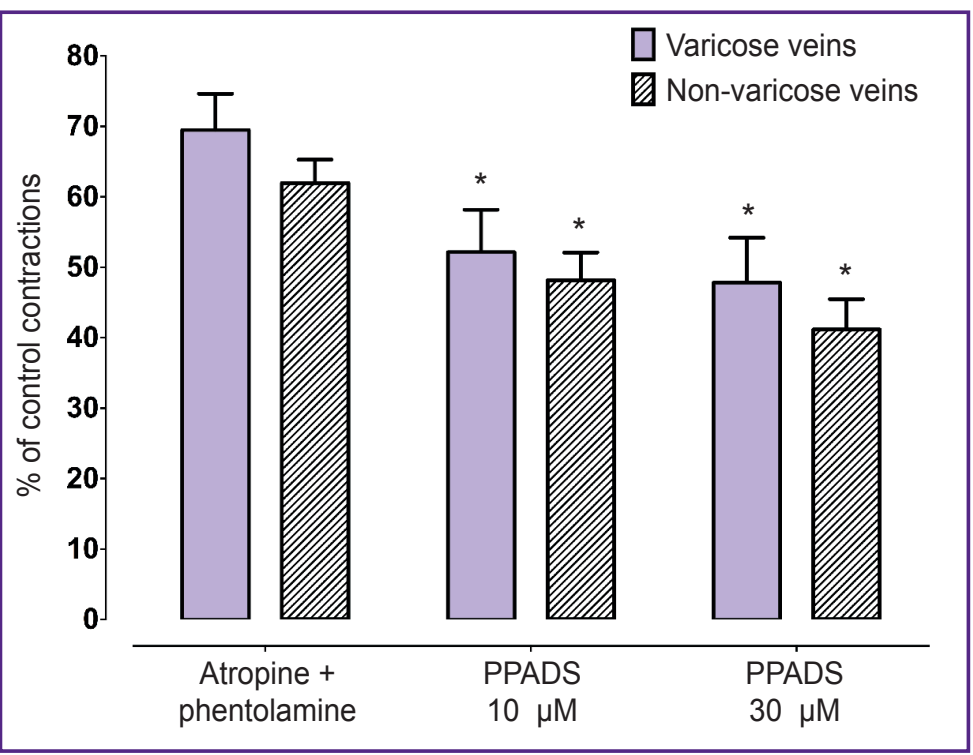

Figure 3. Effect of PPADS (10 and $30 \mu \mathrm{M})$ on the contractile responses of the human great saphenous vein affected by varicose disease $(n=14)$ and without it $(n=21) ; *{ }^{*}<0.05$ when compared to the contractile activity in the presence of atropine and phentolamine 
Figure 4. Effect of suramin (100 and $300 \mu \mathrm{M}$ ) on the contractile responses of the human great saphenous vein affected by varicose disease $(n=14)$ and without it $(n=21) ;{ }^{*} p<0.05$ when compared to the contractile activity in the presence of atropine and phentolamine

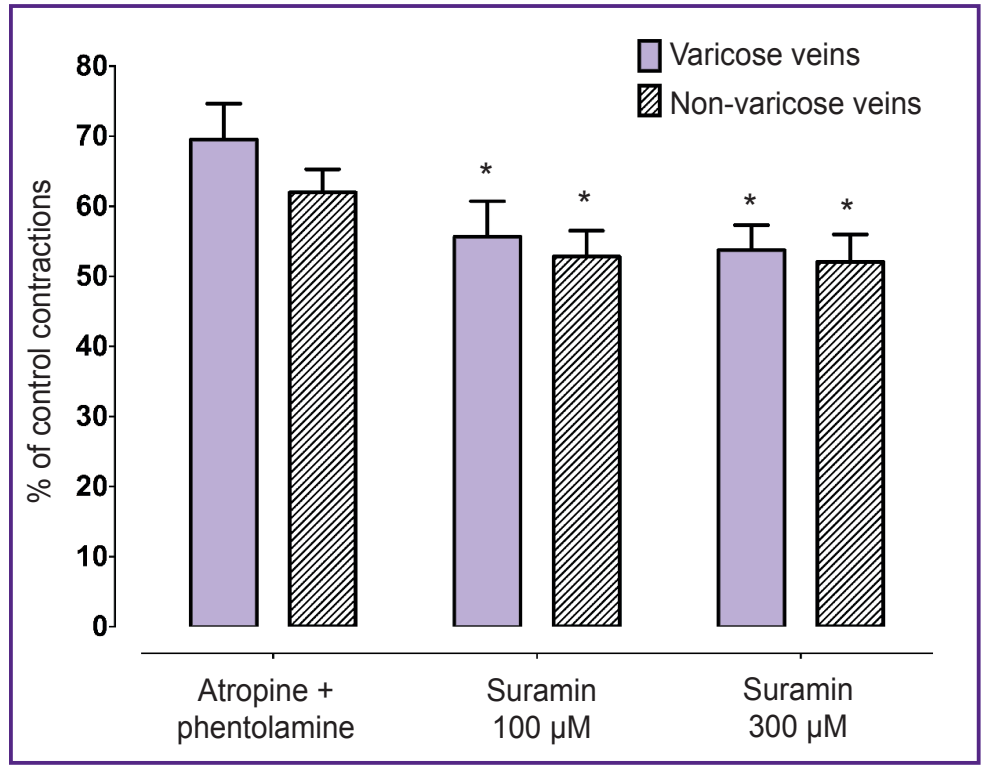

Figure 5. Effect of $\alpha, \beta$-methylene ATP $(10 \mu \mathrm{M})$ on the contractile responses of the human great saphenous vein affected by varicose disease $(n=14)$ and without it $(n=21)$

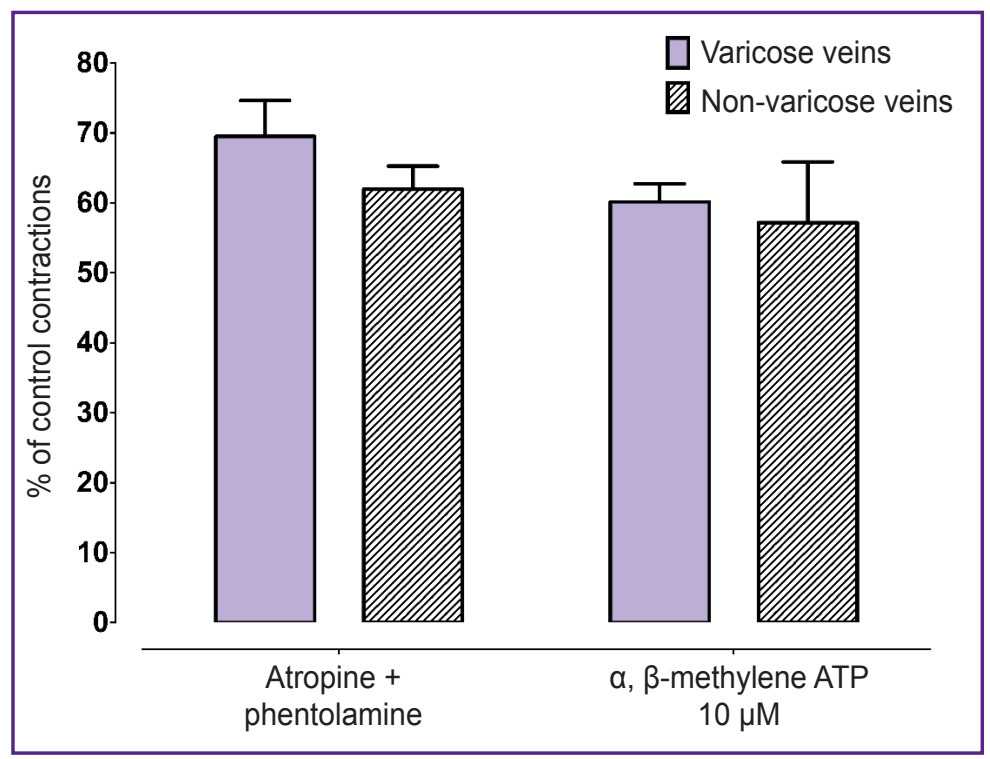

Discussion. This study suggests the presence of nonadrenergic and noncholinergic components of neurotransmission in the GSV. The efficacy of P2 receptor antagonists, PPADS and suramin, allows us to hypothesize that $\mathrm{P} 2$ receptor-mediated component is a part of the nonadrenergic and noncholinergic components of the nervous regulation of the GSV tone.

The presence and activity of $\mathrm{P} 2$ receptors in varicose and non-varicose human GSV have been previously shown by our group [7-9] and other researchers [10]. We found various subtypes of P2 receptors in smooth muscle cells and in the endothelial layer of the GSV [9]. However, the extent of involvement of these receptors in the formation of the GSV tone remained unstudied. The results of our study confirm that P2 receptors play an important role in the neurogenic tone control in both normal and diseased GVS. Furthermore, the detected nonadrenergic and noncholinergic component of neurotransmission suggests the presence of endogenous ligands (such as ATP) that are released, when the nerves are stimulated, and act via P2 receptors. In previous investigations [1, 12], it has been shown that ATP can be released from the nerve terminals (sympathetic and sensomotor) in different human and animal blood vessels together with other neuromediators acting as a co-transmitter. Acting in conjunction with noradrenoline (during mutual exocytosis from sympathetic nerves) in blood vessels ATP most commonly activates the P2X1 subtype of receptors and evokes contraction of the smooth muscle cells. ATP released from sensomotor neuron endings most commonly activates the $P 2 Y_{1,2,6}$ or $P 2 Y_{12}$ receptor subtypes, causing either contraction or relaxation of the blood vessel [1]. 
P2 receptors in the GSV can play several important roles simultaneously. There is a theory that the P2 receptor system is much older in evolution than other receptor systems [13]. Therefore, it may be that the P2 receptor system in human blood vessels yields priority in realization of vasomotor effect to the more evolutionarily advanced receptor systems, e.g. the adrenergic system. At the same time, the role of $\mathrm{P} 2$ receptors significantly increases in pathological conditions such as inflammation, pain, thrombosis and vasospasm $[14,15]$. Thus, P2 receptors play a role of a specific protective mechanism, which may be activated by the human organism during certain pathological processes. In particular, in varicose disease, the P2 receptor apparatus of the GSV undergoes reorganization, which manifests itself in altering the sensitivity of P2 receptors to the action of agonists and antagonists on various segments of the GSV [7]. Such reorganization may signify the involvement of P2 receptors in the pathogenesis of varicose disease development, which to date is not well studied.

The results of the present study have shown that the efficacy of adreno-, cholinoreceptors and $\mathrm{P} 2$ receptors is less in varicose-affected veins in comparison with the veins without varicose disease. Thereby, we hypothesize that in the process of varicose disease development, alterations are likely to occur not only in adreno- and cholinoreceptors, but in the system of P2 receptors as well. Unidirectional involvement of all studied receptor systems towards the reduction of their efficacy may signify the generalized loss of receptormediated responses in varicose veins, which may be explained by the reorganization of the wall of the GSV from the contractile to the synthetic type [16]. No doubt, caution should be exercised regarding interpretation of these data and in regard to the comparisons of varicose and non-varicose veins, since investigations were carried out on the GSV of different localization along the extremity.

P2 receptors are promising targets for the development of drugs that would affect the cardiovascular system [5, 17]. At present, several drugs the mechanism of action of which is based on the interaction with $\mathrm{P} 2$ receptors have been widely used in clinical practice: clopidogrel, ticlopidine, and prasugrel [18]. These drugs are powerful antiplatelet medications that act as antagonists of $P 2 Y_{12}$ receptors located on thrombocytes. Detailed investigation of P2 receptors in GSV will allow researchers to define more exactly their role in the pathogenesis of various diseases of the human venous system (such as varicose disease, thrombophlebitis, etc.) and may create prerequisites for the development of drugs, which would act by activation or inhibition of $\mathrm{P} 2$ receptors.

Thus, data from previous studies and the results of the current investigation make it possible to hypothesize that alteration of $\mathrm{P} 2$ receptor sensitivity may serve as one of the important links in the pathogenesis of varicose disease development in the veins of lower extremities. It is possible that further investigations in this field may help identify novel effective means of treating varicose disease.

Conclusion. The identified role of the P2 receptormediated component in the neurogenic control of the human great saphenous vein tone and further investigation of the $\mathrm{P} 2$ receptor mechanism of action may serve as a perspective foundation for the development of novel drugs the effect of which would be similar to that of $\mathrm{P} 2$ receptor antagonists, and which could be used to prevent graft spasms after aortocoronary bypass.

Study Funding. The work was supported by grant of Russian Foundation for Basic Research No.16-0400101 and was partly performed in the framework of the Russian Government Program of Competitive Growth of Kazan Federal University.

Conflicts of Interest. The authors have no conflicts of interest to disclose.

\section{References}

1. Burnstock G., Ralevic V. Purinergic signaling and blood vessels in health and disease. Pharmacol Rev 2014; 66(1): 102-192, https://doi.org/10.1124/pr.113.008029.

2. Ziganshin A.U., Ziganshina L.E. P2-receptory: perspektivnaya mishen' dlya buduschikh lekarstv [P2 receptors: perspective targets for future drugs]. Moscow: GEOTAR-Media; 2009.

3. Burnstock G. Purine and pyrimidine receptors. Cell Mol Life Sci 2007; 64(12): 1471-1483, https://doi.org/10.1007/ s00018-007-6497-0.

4. Burnstock G. Purinergic regulation of vascular tone and remodelling. Auton Autacoid Pharmacol 2009; 29(3): 63-72, https://doi.org/10.1111/j.1474-8673.2009.00435.x.

5. Ralevic V. P2X receptors in the cardiovascular system and their potential as therapeutic targets in disease. Curr Med Chem 2015; 22(7): 851-865, https://doi.org/10.2174/09298673 21666141215094050.

6. Eklof B., Perrin M., Delis K.T., Rutherford R.B., Gloviczki P. Updated terminology of chronic venous disorders: the VEIN-TERM transatlantic interdisciplinary consensus document. J Vasc Surg 2009; 49(2): 498-501, https://doi. org/10.1016/j.jvs.2008.09.014.

7. Ziganshin B.A., Slavin D.A., Ziganshina A.P., Dzhordzhikiya R.K., Ziganshin A.U. Functional activity of varicose human great saphenous vein at different sites. Bull Exp Biol Med 2013; 155(4): 429-432, https://doi.org/10.1007/ s10517-013-2169-2.

8. Ziganshin A.U., Khaziakhmetov D.F., Ziganshina L.E., Khaziakhmetova V.N., Jourjikiya R.K., Ziganshin B.A., Giniyatova L.R., Burnstock G. Varicose disease affects the P2 receptor-mediated responses of human greater saphenous vein. Vascul Pharmacol 2004; 42(1): 17-21, https://doi. org/10.1016/j.vph.2004.11.007.

9. Ziganshin B.A., Slavin D.A., Khaziakhmetov D.F., Ziganshina A.P., Slavin L.E., Dzhordzhikiya R.K., Ziganshin A.U. Study of the presence and localization of P2 receptors in human blood vessels. Kazanskiy meditsinskiy zhurnal 2015; 96(3): 368-376, https://doi.org/10.17750/ kmj2015-368. 
10. Metcalfe M.J., Baker D.M., Turmaine M., Burnstock G. Alterations in purinoceptor expression in human long saphenous vein during varicose disease. Eur J Vasc Endovasc Surg 2007; 33(2): 239-250, https://doi.org/10.1016/j. jvs.2006.12.035.

11. Eklöf B., Rutherford R.B., Bergan J.J., Carpentier P.H., Gloviczki P., Kistner R.L., Meissner M.H., Moneta G.L., Myers K., Padberg F.T., Perrin M., Ruckley C.V., Smith P.C., Wakefield T.W. Revision of the CEAP classification for chronic venous disorders: consensus statement. J Vasc Surg 2004; 40(6): 1248-1252, https://doi.org/10.1016/j.jvs.2004.09.027.

12. Burnstock $G$. Noradrenaline and ATP as cotransmitters in sympathetic nerves. Neurochem Int 1990; 17(2): 357-368, https://doi.org/10.1016/0197-0186(90)90158-p.

13. Burnstock G., Verkhratsky A. Evolutionary origins of the purinergic signalling system. Acta Physiol (Oxf) 2009; 195(4): 415-447, https://doi.org/10.1111/j.1748-1716.2009.01957.x.
14. Burnstock G., Pelleg A. Cardiac purinergic signalling in health and disease. Purinergic Signal 2015; 11(1): 1-46, https://doi.org/10.1007/s11302-014-9436-1.

15. Ziganshin A.U. Purine P2 receptors as potential targets for action of new drugs. Vestnik Rossiyskoi akademii meditsinskikh nauk 2011; 11: 32-38.

16. Badier-Commander C., Couvelard A., Henin D., Verbeuren T., Michel J.B., Jacob M.P. Smooth muscle cell modulation and cytokine overproduction in varicose veins. An in situ study. J Pathol 2001; 193(3): 398-407, https://doi. org/10.1002/path.819.

17. Ziganshin A.U., Ziganshin B.A. P2 receptors promising targets for future drugs. Current Topics in Pharmacology 2012; 16(1): 31-43.

18. Laine M., Paganelli F., Bonello L. P2Y12-ADP receptor antagonists: days of future and past. World J Cardiol 2016; 8(5): 327-332, https://doi.org/10.4330/wjc.v8.i5.327. 